\section{Jean-Christophe} Renauld

\title{
L'IL9 et son récepteur, un modèle pour l'oncogenèse des lymphocytes T
}

IL9 est une cytokine produite par les lymphocytes T activés. Elle agit sur divers types de cellules, tels que les lymphocytes $\mathbf{T}$ et $\mathrm{B}$, les mastocytes et les précurseurs érythroïdes et myéloïdes, et des lignées neuronales. Son récepteur appartient à la famille des récepteurs hématopoïétiques et transmet le signal à l'intérieur de la cellule en s'associant à la chaîne $\gamma$ du récepteur de l'IL2. Les souris transgéniques exprimant le gène IL9 de manière constitutive développent très souvent des lymphomes thymiques après irradiation ou exposition à un mutagène chimique. Chez l'homme, l'expression constitutive d'IL9 a été trouvée dans des lignées cellulaires et des ganglions lymphatiques provenant de patients atteints de la maladie de Hodgkin ou de lymphomes anaplasiques à grandes cellules.

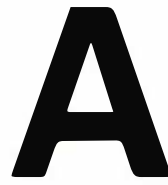
u cours des dix dernières années, les domaines de l'immunologie et de l'hématologie ont été le théâtre de la découverte de nouvelles molécules, les cytokines, caractérisées par de multiples activités in vitro et dont certaines sont déjà l'objet de nombreuses applications in vivo. Comme beaucoup de ces protéines, l'interleukine 9 (IL9) a tout d'abord été décrite sous diverses dénominations avant de prendre sa place dans la nomenclature des interleukines. La première description de cette protéine, réalisée dans notre laboratoire, reposait sur la capacité de surnageants de lymphocytes $\mathrm{T}$ activés de maintenir la croissance de clones de lymphocytes $\mathrm{T}$ auxiliaires murins, en l'absence de cellules accessoires et d'antigène [1]. Cette approche a permis d'obtenir des lignées de lymphocytes $T$ permanentes dont la prolifération dépendait d'un facteur jusqu'alors inconnu. Celui-ci reçut le nom de «P40" sur la base de sa taille, estimée à partir d'expériences de fractionnement sur gel.

Indépendamment, un nouveau facteur de croissance pour les mastocytes avait été partiellement purifié à partir de surnageants de lymphocytes T murins par une équipe allemande [2]. Ce facteur provisoirement dénommé MEA (mast cell growth enhancing activity) était en fait identique à un facteur de croissance pour des lymphocytes $\mathrm{T}$ baptisé TCGF-3 


\section{RÉFÉRENCES}

1. Uyttenhove C, Simpson RJ, Van Snick J. Functional and structural characterization of P40, a mouse glycoprotein with T-cell growth factor activity. Proc Natl Acad Sci USA $1988 ; 85: 6934-8$.

2. Hültner L, Druez C, Moeller J, Uyttenhove $C$, Schmitt $E$, Rüde $E$, Dörmer $P$, Van ve C, Schmitt E, Rüde E, Dörmer P, Van (MEA) is structurally related and functionally identical to the novel mouse $T$ cell growth factor P40/TCGFIII (interleukin-9) Eur J Immunol 1990; 20: 1413-6.

3. Moeller J, Hültner L, Schmitt E, Breuer $M$, Dörmer $P$. Purification of MEA, a mas cell growth-enhancing activity, to apparent homogeneity and its partial amino acid sequencing. J Immunol 1990; 144 : 4231-4.

4. Renauld JC, Goethals A, Houssiau F, Van Roost E, Van Snick J. Cloning and expression of a cDNA for the human homolog of mouse $\mathrm{T}$ cell and mast cell growth factor P40. Cytokine 1990; 2: 9-12.

5. Yang YC, Ricciardi S, Ciarletta A, Calvetti J, Kelleher K, Clark SC. Expression cloning of a cDNA encoding a novel human hematopoietic growth factor: human homologue of murine T-cell growth factor P40. Blood $1989 ; 74: 1880-4$.

6. Van Snick J, Goethals A, Renauld JC, Van Roost E, Uyttenhove C, Rubira MR, Morit RL, Simpson RJ. Cloning and characterization of a cDNA for a new mouse T cell growth factor $(\mathrm{P} 40)$. J Exp Med 1989; 169: 363-8.

7. Renauld JC, Goethals A, Houssiau F, Van Roost E, Van Snick J. Human P40/IL-9 expression in activated CD4 ${ }^{+} \mathrm{T}$ cells, genomic organization and comparison with the mouse gene. J Immunol 1990; 144 : 4235-41.

8. Houssiau F, Renauld JC, Fibbe WE, Van Snick J. IL-2 dependence of IL-9 expression in human T lymphocytes. I Immunol 1992; 148: $3147-51$

9. Druez C, Coulie P, Uyttenhove C, Van Snick I. Functional and biochemical characterization of mouse P40/IL-9 receptors. J Immunol 1990; 145: 2494-9.

10. Renauld JC, Druez C, Kermouni A, Houssiau F, Uyttenhove C, Van Roost E, Van Snick J. Expression cloning of the murine and human interleukin-9 receptor cDNAs. Proc Natl Acad Sci USA 1992;89. 5690-4.

11. Dusanter-Fourt I, Mayeux P, Gisselbrecht $\mathrm{S}$. Transduction du signal par les récepteurs de cytokines. médecine/sciences 1994; 10 : 825-35.

12. Bazan JF. A novel family of growth factor receptors: a common binding domain in the growth hormone, prolactin, erythropoietin and IL -6 receptors, and the p75 IL-2 receptor $\beta$-chain. Biochem Biophys Res Commun 1989; 164: 788-95.

13. Kimura $Y$, Takeshita $T$, Kondo $M$, Ishi N, Nakamura M, Van Snick J, Sugamura K. Sharing of the IL-2 receptor $\gamma$ chain with the functional IL-9 receptor complex. Int
( $T$ cell growth factor 3). A l'instar de P40, le TCGF-3 était décrit comme un facteur de croissance produit par un clone de lymphocyte $\mathrm{T}$ auxiliaire murin actif sur d'autres clones $\mathrm{T}$ [3]. Le clonage de l'ADNc correspondant à la protéine $\mathrm{P} 40$ permit de démontrer que celle-ci était responsable des activités MEA et TCGF-3.

L'équivalent humain de P40 fut lui aussi caractérisé par deux approches différentes. D'une part, l'utilisation de l'ADNc murin nous conduisit à identifier, par hybridation croisée, un ADNc humain codant pour une protéine active sur des lignées de lymphocytes T humains [4]. D'autre part, ce même ADNc était isolé dans le cadre du clonage d'une nouvelle protéine humaine induisant la prolifération in vitro d'une lignée de leucémie mégacaryoblastique [5]. C'est d'ailleurs à la suite de cette observation qui suggérait un rôle pour P40 dans l'hématopoïèse que cette molécule fut baptisée IL9.

Depuis lors, le spectre d'activité de l'IL9 s'est encore étendu et comprend la production d'immunoglobulines par les lymphocytes B, la prolifération des précurseurs hématopoïétiques de la lignée rouge, les thymocytes fotaux et même la différenciation de lignées neuronales murines. En outre, une série de résultats qui seront discutés ici suggèrent que cette cytokine pourrait jouer un rôle dans le développement de certains lymphomes chez l'homme et chez la souris.

\section{Clonage et expression de I'IL9}

L'obtention de lignées de lymphocytes T dépendants de IL9 a permis la purification de la protéine naturelle murine à partir de surnageants de lymphocytes T auxiliaires activés [1]. Cette protéine est caractérisée par une glycosylation très abondante. En effet, alors que la protéine purifiée apparaît en gel comme une molécule de masse hétérogène variant entre 32 et $39 \mathrm{kDa}$, un traitement par la N-glycanase réduit celle-ci à 15-16 kDa. La séquence de l'ADNc murin [6] nous a montré que la protéine mûre consiste en un polypeptide de 126 acides aminés auxquels s'ajoute un peptide signal de 18 résidus. Conformément aux estimations faites après déglycosylation, la masse molaire prédite par la séquence est de $14150 \mathrm{Da}$ et la présence de quatre sites potentiels de glycosylation explique vraisemblablement la différence de masse molaire avec la protéine native.

L'IL9 humaine naturelle n'a jamais été purifiée, l'ADNc ayant été directement identifié, soit par hybridation croisée avec son homologue murin, soit à la suite du clonage par expression d'un facteur induisant la prolifération d'une lignée cellulaire dérivée d'une leucémie mégacaryoblastique humaine. La protéine humaine possède le même nombre d'acides aminés dont $55 \%$ sont identiques à ceux de la protéine murine.

Nous avons caractérisé le gène de l'IL9 chez l'homme et chez la souris. Il est constitué de 5 exons et 4 introns qui s'étendent sur un peu plus de $3 \mathrm{~kb}$ [7]. Chez l'homme, ce gène est localisé sur le chromosome 5 , dans la région 5q31.1-q33. Cette région du génome se caractérise par la présence de nombreux gènes de cytokines ou de récepteurs de cytokines (figure 1) et semble être impliquée dans certaines maladies. En effet, des délétions dans cette région sont fréquemment observées dans des syndromes de myélodysplasie, tels que l'anémie réfractaire, qui sont susceptibles de dégénérer en leucémies aiguës. En outre, il a été démontré récemment que cette région du génome est impliquée dans la régulation de la production d'IgE, les anticorps responsables de nombreux phénomènes allergiques.

L'expression de l'IL9 semble être limitée aux lymphocytes $\mathrm{T}$ activés. Chez la souris, l'IL9 est produite par les lymphocytes $\mathrm{T}$ auxiliaires du type Th2. Ceux-ci produisent également de l'IL4 et de l'IL5, mais pas d'interféron $\gamma$. Il a également été montré que des réponses immunitaires qui induisent une activation de ces lymphocytes Th2 in vivo, comme certaines infections parasitaires, s'accompagnent d'une forte production d'IL9. Chez l'homme, l'IL2 a été identifiée comme l'un des relais intervenant dans l'expression de l'IL9 par les lymphocytes $T$ activés [8]. En effet, celle-ci est complètement bloquée par un anticorps dirigé contre le récepteur de l'IL2. L'IL2 n'est en fait que la première étape d'une cascade de cytokines 


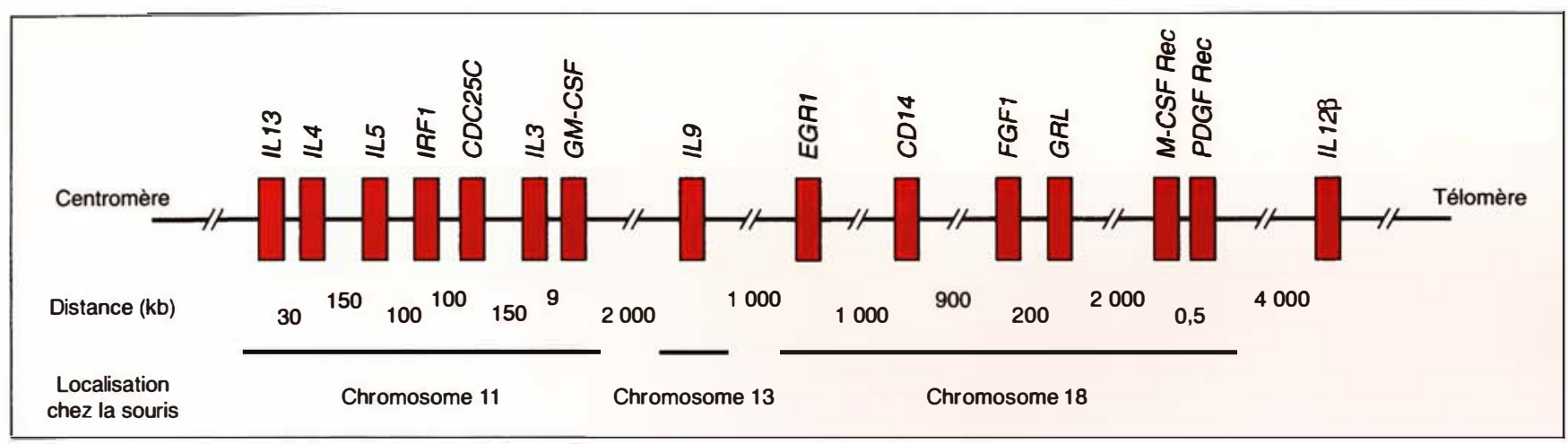

Figure 1. Représentation schématique de la région du chromosome 5 humain qui comprend le gène de l'IL9. Les rectangles correspondent aux gènes codant pour les protéines suivantes: interleukines 13, 4, 5, 3, 9, 12- $\beta$ (IL 13, 4, 5, 3, 9, 12- $\beta$ ), interferon regulatory factor 1 (IRF1), cell division cycle 25 (CDC25C), granulocyte-macrophage colony stimulating factor (GM-CSF), early growth factor response 1 (EGR1), cluster of differentiation antigen 14 (CD14), fibroblast growth factor 1 (FGF1), glucocorticoid receptor lymphocyte-specific (GRL), macrophage colony stimulating factor receptor (M-CSF Rec), platelet derived growth factor receptor (PDGF Rec).

puisqu'elle contrôle la production de l'IL4 qui, elle-même, contrôle la production de l'IL10. L'IL4 et l'IL10 sont toutes deux indispensables à la production d'IL9.

\section{Le récepteur de I'IL9}

Les caractéristiques fonctionnelles et biochimiques du récepteur de l'IL9 murine ont été étudiées dans notre laboratoire en utilisant l'IL9 recombinante produite dans des cellules d'insectes. Des sites de fixation spécifiques de l'IL9 ont été détectés sur des cellules d'origines diverses comme des clones $\mathrm{T}$ auxiliaires, des lignées de mastocytes, de macrophages et de thymomes [9]. L'analyse selon Scatchard de ces sites de fixation présents à la surface d'un clone de lymphocytes $\mathrm{T}$ a montré qu'ils appartiennent à une seule classe de forte affinité (Kd $\sim 100 \mathrm{pM})$.

Nous avons identifié l'ADNc du récepteur de l'IL9 murine par une méthode de clonage par expression directe dans les cellules COS (cellules épithéliales de rein de singe) [10]. L'expression de cet ADNc dans les cellules COS, normalement dépourvues de récepteur de l'IL9, entraîne l'apparition de sites de fixation caractérisés par une affinité comparable à celle du récepteur présent à la surface des lymphocytes $\mathrm{T}$. Le clonage du récepteur de l'IL9 murine a, par ailleurs, permis l'identification du récepteur humain par $m / s n^{\circ} 9$, vol. 11 , septembre 95 hybridation croisée. Ce récepteur est une protéine de 468 acides aminés chez la souris et de 522 acides aminés chez l'homme, $55 \%$ étant identiques entre les protéines humaine et murine. Il s'agit d'une protéine ayant un domaine transmembranaire unique, un domaine extracellulaire de 234 acides aminés et un domaine cytoplasmique de 177 acides aminés chez la souris et 231 chez l'homme [10]. Le domaine extracellulaire du récepteur de l'IL9 est non seulement le domaine le mieux conservé entre l'homme et la souris, mais il possède en outre une analogie significative avec plusieurs autres récepteurs de cytokines comme les récepteurs de l'IL4, de l'IL6, de l'IL7, etc. En particulier, on retrouve plusieurs cystéines à des distances précises ainsi qu'un motif WSEWS situé à proximité de la région transmembranaire et caractéristique de cette nouvelle famille des récepteurs hématopoïétiques [11, 12]. $\mathrm{Au}$ contraire de la région extracellulaire, le domaine cytoplasmique ne possède pas d'analogie significative avec d'autre récepteurs, à l'exception de la chaîne $\beta$ du récepteur de l'IL2 dans la partie située immédiatement en aval de la région transmembranaire. Les mécanismes responsables de la transmission du signal par le récepteur de l'IL9 restent encore mal connus. Des expériences récentes [13] ont montré que ce récepteur s'associe à une protéine transmembranaire appelée $\gamma_{c}$ et qui est égale- ment impliquée dans le fonctionnement des récepteurs de l'IL2, de l'IL4 et de l'IL7 (figure 2). En effet, un anticorps dirigé contre $\gamma c$ est capable d'inhiber la réponse à l'IL9 sans pour autant interférer avec sa fixation sur les cellules. Cette protéine $\gamma c$ représente ainsi une des clefs de l'activation des lymphocytes. En effet, des mutations dans le gène qui code pour $\gamma_{c}$ sont responsables de syndromes d'immunodéficience sévères chez l'homme [11].

\section{Activités biologiques de I'IL9}

Les activités biologiques de l'IL9 dans des modèles expérimentaux in vitro sont résumées dans la figure 3 et concernent essentiellement les lymphocytes $\mathrm{T}$, les mastocytes, les lymphocytes $B$, les précurseurs hématopoiétiques, et les cellules neuronales. Contrairement à d'autres facteurs de croissance des lymphocytes $\mathrm{T}$ comme l'IL2 ou l'IL4, l'IL9 semble être dépourvue d'activité sur des lymphocytes $\mathrm{T}$ fraîchement isolés. Chez l'homme, la capacité de répondre à l'IL9 semble exiger une préactivation des cellules et peut être obtenue en stimulant les lymphocytes in vitro avec des lectines, de l'IL2 et des cellules allogéniques [14]. La seule activité de l'IL9 observée directement sur des lymphocytes $\mathrm{T}$ frais concerne la prolif ération de thymocytes fotaux murins en synergie avec l'IL2 [15], 


\section{RÉFÉRENCES}

14. Houssiau F, Renauld JC, Stevens M, Lehmann $F$, Lethe B, Coulie PG, Van Snick J. Human $T$ cell lines and clones respond to IL-9. J Immunol 1993; 150: 2634-40.

15. Suda T, Murray R. Fischer M, Yokota T, Zlotnik A. Tumor necrosis factor- $\alpha$ and P40 induce day 15 murine fetal thymocyte proliferation in combination with IL-2. I Immunol 1990; 144: 1783-7.

16. Dugas B, Renauld JC, Pene J, Bonnefoy JY, Petit-Frère $C$, Braquet $P$, Bousquet J, Van Snick J, Mencia-Huerta JM. Interleukin-9 potentiates the interleukin-4-induced immunoglobulin (IgG, $\operatorname{IgM}$ and $\operatorname{IgE}$ ) production by normal human $B$ lymphocytes Eur J Immunol 1993; 23: 1687-92.

17. Petit-Frère $C$, Dugas $B$, Braquet $P$, Men cia-Huerta JM. Interleukin-9 potentiates the interleukin-4-induced IgE and IgGl release from murine B lymphocytes. Immunology 1993: $79: 146-51$.

18. Donahue RE, Yang YC, Clark SC. Human P40 T-cell growth factor (interleukin-9) supports ervthroid colony formation. Blood 1990; 75: 2271-5.

19. Holbrook ST, Ohls RK, Schribler KR, Yang YC, Christensen RD. Effect of interleukin-9 on clonogenic maturation and cellcycle status of fetal and adult hematopoiesis progenitors. Blood 1991; 77: 2129-34.

20. Mehler MF, Rozental R, Dougherty M Spray DC, Kessler JA. Cytokine regulation of neuronal differentiation of hippocampal progenitor cells. Nature 1993; 362: 62-5.

21. Uyttenhove C, Druez C, Renauld JC, Herin M, Noel H, Van Snick J. Autonomous growth and tumorigenicity induced by P40/interleukin 9 cDNA transfection of a mouse P40-dependent $T$ cell line. $J$ Exp Med 1991; 173: 519-22.

22. Vink A, Renauld JC, Warnier G, Van Snick J. Interleukin-9 stimulates in vitro growth of mouse thymic lymphomas. Eur J Immunol 1993; $23: 1134-8$.

23. Renauld JC, Vink A, Louahed J, Van Snick J. IL-9 is a major anti-apoptotic facto for thymic lymphomas. Blood $1995 ; 85: 1300$ 5.

24. Renauld JC, van der Lugt N, Vink A, van Roon M, Godfraind C, Warnier G, Merz H Feller A, Berns A, Van Snick J. Thymic lymphomas in interleukin-9 transgenic mice. Oncogene 1994; 9 : 1327-32.

25. Merz H, Houssiau F, Orscheschek K Renauld JC, Fliedner A, Herin M, Noël $H$, Kadin M, Mueller-Hermelink H, Van Snick J, Feller AC. Interleukin-9 expression in human malignant lymphomas: unique association with Hodgkin's disease and large cell anaplastic lymphoma. Blood 1991; 78 $1311-7$.

26. Gruss HJ, Brach MA, Drexler HG, Bross $\mathrm{KJ}$, Herrmann F. Interleukin 9 is expressed by primary and cultured Hodgkin and Reed-Sternberg cells. Cancer Res 1992; 52 1026-31.

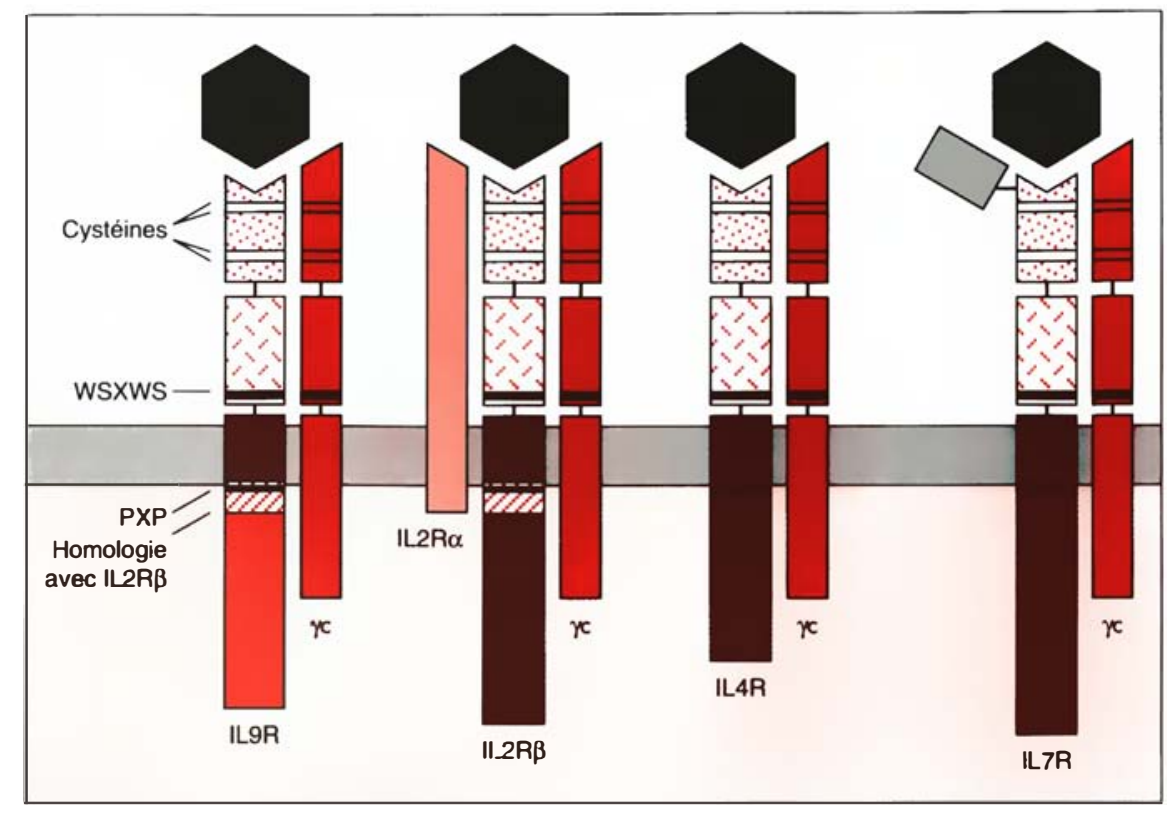

Figure 2. Utilisation d'une chaîne commune $(\gamma c)$ par les récepteurs de I'IL2, de I'IL4, de I'IL7 et de I'IL9. Le domaine extracellulaire de toutes ces chaines est caractérisé par deux paires de cystéines dans la région $\mathbf{N}$-terminale, engagées dans des ponts disulfure intrachaînes, nécessaires à la liaison du ligand, et un motif WSXWS (trp-ser-acide aminé quelconque-trp-ser). Le domaine cytoplasmique d'IL9 ne présente pas d'analogie avec $d$ 'autres récepteurs, à l'exception de la chaîne $\beta$ du récepteur de I'IL2, immédiatement en aval de la région transmembranaire. Le motif PXP (deux prolines de part et $d^{\prime}$ autre $d^{\prime} u n$ acide aminé variable) est une caractéristique du domaine cytoplasmique de la plupart des récepteurs hématopoiétiques.

alors que l'IL9 semble inactive sur les thymocytes adultes.

Les mastocytes représentent certainement la cible privilégiée de l'IL9. Elle stimule en effet la prolifération de lignées mastocytaires murines, soit seule, soit en synergie avec l'IL3 ou avec le steel factor [2]. En outre, l'IL9 est capable d'induire la différenciation de ces cellules in vitro. Elle induit notamment l'expression de toute une série de protéases spécifiques des granules des mastocytes, la production d'IL6 ainsi que l'expression du récepteur de forte affinité pour les IgE par ces lignées mastocytaires. Cette activité de l'IL9 a été confirmée in vivo chez des souris transgéniques pour l'IL9 qui montrent une mastocytose systémique massive. L'IL9 semble jouer un rôle particulièrement important dans le développement de réponses mastocytaires comme celles induites par les infections parasitaires intestinales. Les lymphocytes B représentent également une cible physiologique de
l'IL9. Elle y potentialise l'effet de l'IL4 sur la production d'IgG et d'IgE, in vitro, dans des modèles expérimentaux murins et humains $[16,17]$ ainsi que, in vivo, chez les souris transgéniques pour l'IL9 qui produisent des taux anormalement élevés d'immunoglobulines.

Dans le système hématopoïétique adulte, l'IL9 induit, in vitro, le développement de colonies de la lignée des globules rouges en présence d'érythropoïétine et semble avoir un spectre d'activités relativement limité aux précurseurs de la lignée érythroïde [18]. Chez le fotus, l'IL9 est non seulement plus active sur les précurseurs érythroïdes mais est aussi capable d'induire la maturation de colonies d'autres lignées (CFU-Mix et CFU-GM) [19].

Enfin, certaines expériences suggèrent que les cytokines en général, et l'IL9 en particulier, influencent la différenciation de cellules neuronales et sont impliquées dans le développement du système nerveux [20]. 


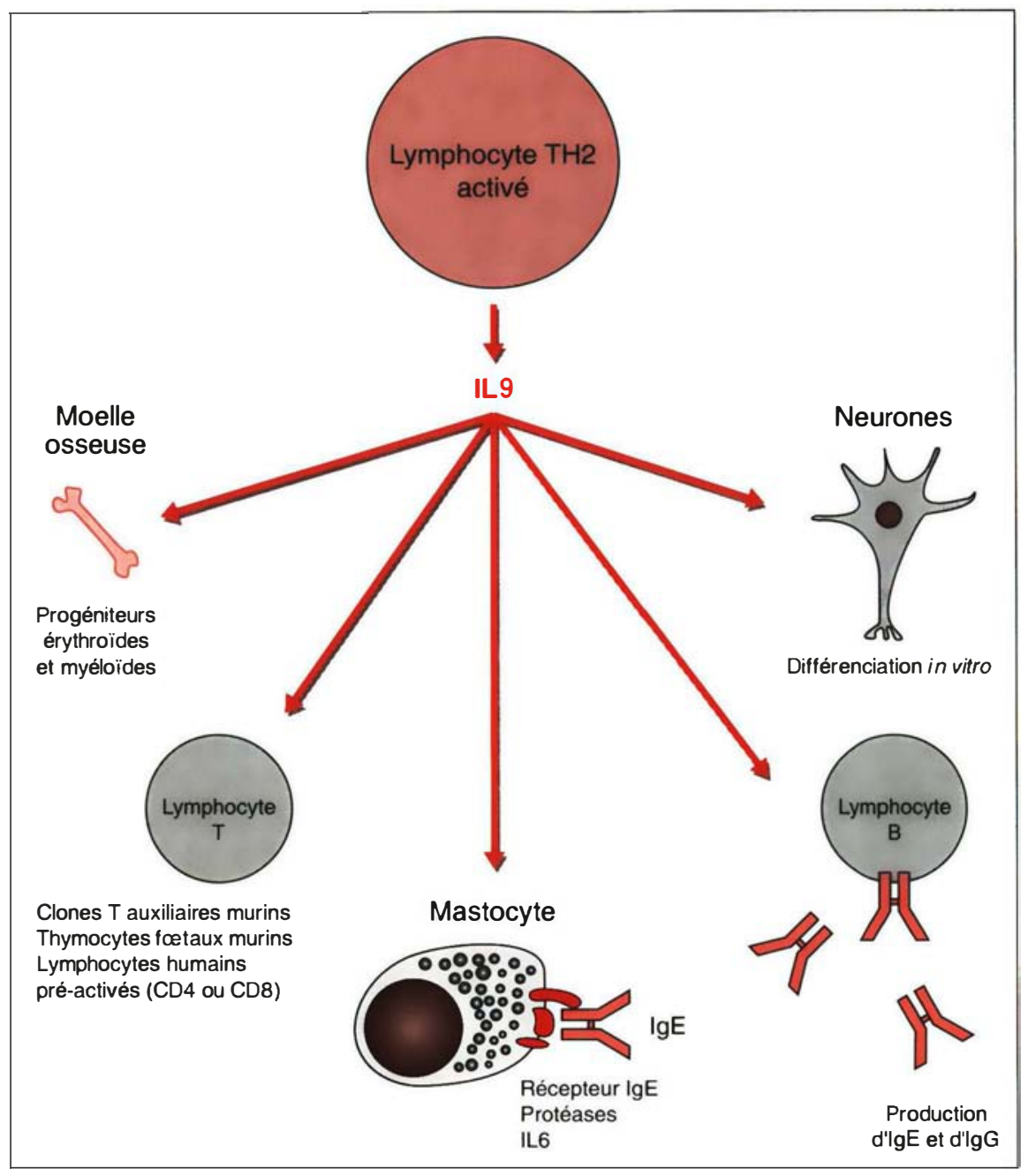

Figure 3. Cibles biologiques de I'IL9 in vitro. IL9 stimule la prolifération des mastocytes, soit seule, soit en synergie avec l'IL3 et le steel factor. En outre, elle semble capable d'induire leur différenciation (synthèse de protéases, IL6 et récepteur des IgE). IL9 ne stimule la prolifération des lymphocytes $T$ que si ceux-ci sont préalablement activés là l'exception des clones $T$ auxiliaires murins et des thymocytes murins fotaux). En revanche, les lymphocytes $B$ représentent une cible pour IL9 qui potentialise I'effet de IL4 sur la production $d^{\prime} \operatorname{lgE}$ et $d^{\prime} \lg G$. IL9 induit la prolifération des progéniteurs érythroïdes chez l'adulte et tous les progéniteurs myéloïdes chez le fotus. Enfin, comme $d^{\prime}$ autres cytokines, IL9 influence la différenciation des cellules neuronales in vitro et semble impliquée dans le développement du système nerveux.

\section{Rôle de I'IL9 dans l'oncogenèse des lymphocytes $T$}

Le paradoxe de l'IL9 réside incontestablement dans le fait que cette protéine, initialement décrite comme un facteur de croissance pour certains clones de lymphocytes $\mathrm{T}$, semble totalement dépourvue d'activité sur des lymphocytes $\mathrm{T}$ fraîchement iso- capable de répondre à l'IL9 à la suite de longues périodes de culture in vitro et que ce phénotype s'accompagne d'une transformation cryptique vers un comportement de cellule tumorale. La figure 4 résume de façon schématique les différentes étapes de cette transformation. Les clones de lymphocytes $\mathrm{T}$ auxiliaires sont généralement dérivés à partir de ganglions lymphatiques de souris immunisées contre un antigène, en restimulant les cellules lymphoïdes en présence de cet antigène et de cellules présentatrices irradiées. A l'issue de ces stimulations, la réponse proliférative aux cytokines des lymphocytes T peut être testée. Dans les premières semaines de culture, on n'observe généralement pas de réponse à l'IL9. Après un peu plus de deux mois, on observe que l'IL9 induit une survie de ces cellules puis, quelque temps plus tard, une réelle prolifération. A ce stade, il est possible de remplacer dans la culture l'antigène et les cellules accessoires par l'IL9 et on obtient ainsi des lignées de lymphocytes $\mathrm{T}$ dont la prolifération à long terme dépend uniquement de ce facteur de croissance. Ces cellules possèdent déjà un potentiel tumoral. En effet, lorsqu'elles sont transfectées avec l'ADNc de l'IL9, non seulement elles deviennent capables de proliférer de façon autonome in vitro, mais elles se développent in vivo sous forme de lymphomes lorsque qu'elles sont injectées à des souris syngéniques [21]. En outre, ce stade de cellules autonomes est sporadiquement acquis par quelques cellules de la lignée dépendante de l'IL9 qui accomplissent ainsi, in vitro, un cycle complet de transformation tumorale. Indépendamment, il a été démontré que l'IL9 est un facteur de croissance particulièrement actif sur les lignées de lymphomes thymiques in vitro [22], alors que les thymocytes normaux ne semblent pas répondre à l'IL9. Enfin, nous avons montré récemment qu'outre son activité de facteur de croissance, l'IL9 exerce sur les cellules de lymphomes thymiques une activité de protection contre l'apoptose induite par la dexaméthasone [23].

L'importance de ces observations a été renforcée par l'analyse de souris transgéniques pour l'IL9, qui expri- lés. En fait, les lymphocytes T peuvent acquérir la capacité de répondre à l'IL9 et cela essentiellement par propriété peut s'acquérir parallèlecomme cela a été démontré dans un modèle humain [14]. D'autre part, les modèles murins ont montré que des clones de lymphocytes $\mathrm{T}$ auxiliaires deviennent progressivement 


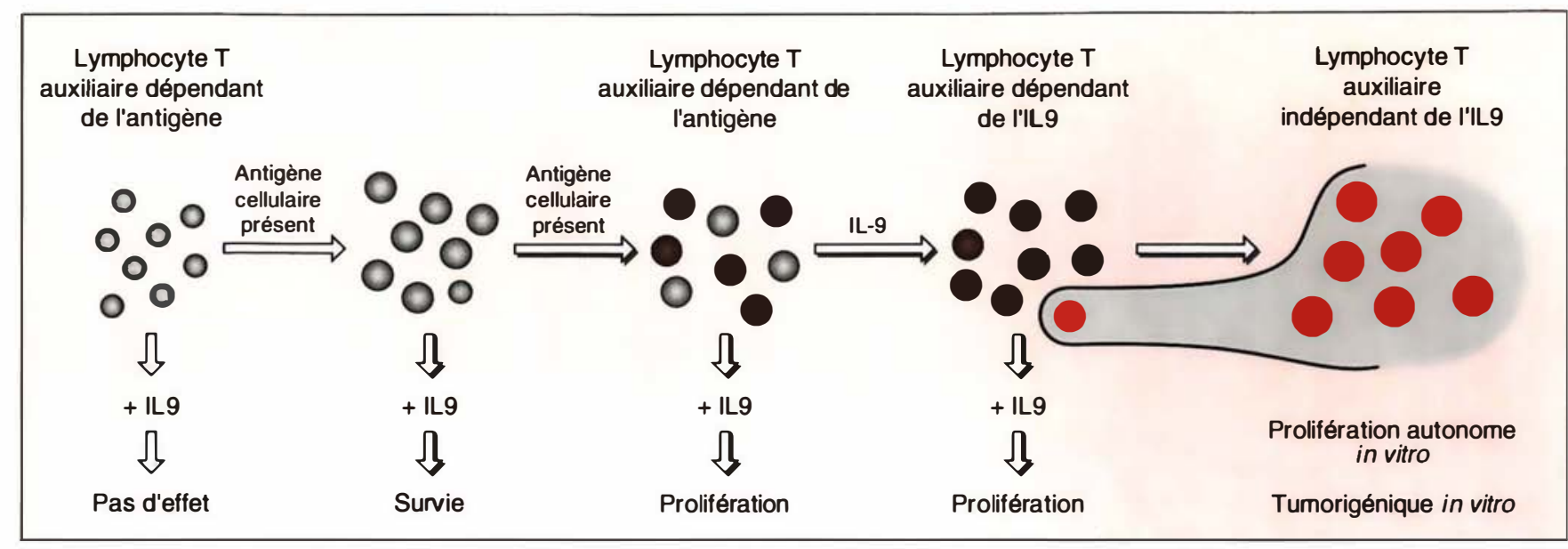

Figure 4. L'acquisition par les lymphocytes T murins de la capacité de répondre à l'llg s'accompagne d'un phénomène de transformation tumorale in vitro. Deux mécanismes peuvent permettre aux lymphocytes $T$ de répondre à I'IL9: I'activation des cellules et la transformation tumorale survenant après de longues périodes de culture. Les clones de lymphocytes $T$ auxiliaires dérivés de ganglions lymphatiques de souris immunisées contre un antigène ne sont pas sensibles à I'IL9 pendant plusieurs semaines; après environ deux mois, IL9 induit une survie de ces cellules puis leur prolifération. Si, à ce stade, on remplace dans la culture l'antigène par IL9, on obtient des lignées de cellules $T$ dépendantes de I'IL9 qui ont déjà un potentiel tumoral. Elles se développent in vivo sous forme de lymphomes lorsqu'on les injecte à des souris syngéniques.

ment de façon constitutive de grandes quantités de cette cytokine. En effet, alors que la plupart des souris transgéniques semblent peu affectées par la surexpression d'IL9, on observe, chez $5 \%$ à $10 \%$ des animaux, l'apparition de lymphomes thymiques envahissant le thymus, la rate et les ganglions lymphoïdes. L'incidence des tumeurs peut même augmenter jusqu'à $100 \%$ après une légère irradiation des souris ou après injection de doses très faibles d'un agent mutagène, ce qui montre que la dérégulation de la production d'IL9 induit une plus grande susceptibilité au développement de ce type de lymphome [24].

Chez l'homme, une expression constitutive d'IL9 a été démontrée dans les ganglions de patients atteints de lymphome de Hodgkin ou de lymphome anaplasique à grandes cellules [25]. L'hypothèse d'une boucle autocrine dans certaines de ces tumeurs a en outre été démontrée pour une lignée de lymphome de Hodgkin dont la prolifération in vitro est inhibée par des anticorps dirigés contre l'IL9 ou des oligonucléotides antisens [26]. Ces résultats deman- étendus avant de commencer à entrevoir certaines applications thérapeutiques. Les années à venir devraient voir se préciser l'importance physiologique de cette cytokine

\section{TIRÉS À PART}

J.C. Renauld.

\section{Summary}

IL9 and its receptor: involvement in $T$ cell oncogenesis.

IL9 is a cytokine produced by activated $\mathrm{T}$ cells and particularly from the Th2 subset. This protein shows pleiotropic activities on various cell types such as $\mathrm{T}$ and B lymphocytes, mast cells, erythroid and myeloid precursors and neuronal cell lines. The IL9 receptor is a member of the hematopoietin receptor superfamily and associates with the $\gamma$ chain of the IL2 receptor to transduce the signal inside the cells. IL9 transgenic mice, constitutively expressing large amounts of this cytokine, are highly susceptible to the development of thymic lymphomas after irradiation or injection of a chemical mutagen. In the human, constitutive IL9 expression has been found in cell lines and lymph nodes from patients with Hodgkin disease or large cell anaplastic lymphomas and an IL9-mediated autocrine loop has been demonstrated for one Hodgkin cell line in vitro. 